\title{
Vibration Analysis of a Spring Supported FG Beam Under Harmonic Force
}

\author{
Cihan Demir and Merter Altinoz \\ Department of Mechanical Engineering, Faculty of Mechanical Engineering, Yildiz Technical University, Turkey.
}

(Received 19 March 2017; accepted 2 February 2018)

The harmonic response and resonance frequency behavior of the spring supported FGM beam against the lateral motion for the various values of support stiffness at the ends are analyzed by using the force transmissibility parameter with the finite-element method and Lagrange's equations. The steady-state responses to a sinusoidal varying force are determined for the support reactions in a frequency domain. The problem is solved within the framework of the Timoshenko beam theory. For the convergence study, some of the results are compared with previous works' values, and a good agreement can be seen. The study concludes that the support stiffness, volume fraction coefficient and the ratio of young module level all strongly affect the natural frequencies and the force transmissibility. For some values of spring stiffness of support, the rigid body motion of the beam occurs and reduces the effect of volume fraction coefficient on the natural frequencies.

\section{INTRODUCTION}

Pure metal usage is limited in engineering application since the application may be in need of a material which has almost opposite properties. For instance, there may be a material requirement for an engineering application, like a material having both a good strength and a temperature resistance. The alloys serve that purpose to a certain extent but they have some limitations varying by their production method, like the thermodynamic equilibrium limit, the difficulty of alloying the two different materials having melting points quite a change. The composite structures can also combine two materials having different properties, but they have a disadvantage too. When two materials constituting the composite structure have a different coefficient of expansion, separation at the interface of the composite can be seen in high temperature.

In order to overcome the problems above, especially the problem of composite structure, in a space plane project, Japan scientists came up with an idea of functionally graded material (FGM) in 1984 during. Since material and mechanical properties of FGM vary gradually from one surface to another, it cannot be seen as a separation. Despite the difficulty in production of FG material, the wide range of application areas and above- mentioned advantages drive the researchers to study FGM structures like beams, plates and shells in the last decade. Sankar studied the elasticity solution of the functionally graded beams subjected to transverse loading 1with the assumption of Euler-Bernoulli beam theory. ${ }^{1}$ The material properties are assumed to vary exponentially through the thickness. He showed that the beam theory is valid for long, slender beams with slowly varying transverse loading. Aydogdu and Taskin investigated the free vibration of simply supported functionally graded beams by using various beam theories. ${ }^{2}$ The beam theories that they used to investigate the free vibration of the simply supported FG beam are Euler-Bernoulli beam theory, parabolic shear deformation beam theory (PSDBT) and ex- ponential shear deformation beam theory (ESDBT). Material property variation through the thickness is considered as the power law and exponential law.

Simsek and Kocaturk investigated the free and forced vibration of a functionally graded simply-supported beam subjected to a concentrated moving harmonic load. ${ }^{3}$ The material properties are assumed to vary through the thickness according to the power-law method. The Euler-Bernoulli beam theory is used while driving equations. Su et al. developed the dynamic stiffness method to investigate the free vibration behavior of functionally graded beams. ${ }^{4}$ They derived the governing differential equations of motion and natural boundary conditions for free vibration by using Hamilton's principle. A parametric study is carried out to demonstrate the effects of the length to thickness ratio and the variation of the power law index parameter. Suddoung et al. investigated the free vibration response of the stepped beams made from functionally graded materials for the various types of elastically end constraints. ${ }^{5}$ The differential transformation method (DTM) is employed to obtain natural frequencies and mode shapes of stepped beam. The step ratio, step location, boundary conditions, spring constants and material volume fraction values were taken into investigation parametrically. Li proposed a unified approach for analyzing the static and dynamic behavior of functionally graded beams with the rotary inertia and shear deformation. ${ }^{6}$ Primarily, Timoshenko beam theory is considered. Euler-Bernoulli beam theory is obtained by reducing from the Timoshenko beam theory. The material properties are assumed to vary through the thickness according to the power-law. Yu and Zhong presented a general two-dimensional solution for a cantilever functionally graded beam with arbitrary graded variations of material property distribution in terms of the Airy stress function. ${ }^{7}$ Kapuria et al. proposed a finite element model for the static and free vibration analysis of the layered functionally graded beams by using third order zigzag theory. ${ }^{8}$ They estimated the effective modulus of elasticity. It is also validated by experiments for 
two different functionally graded beam systems under different boundary conditions. Piovan and Sampaio performed the study of the vibrations of functionally graded sliding beams by considering the Euler-Bernoulli beam theory with finite element modeling. ${ }^{9}$ The authors developed this study for functionally graded rotating beams. Sina et al. developed a new beam theory to analyze the free vibration of functionally graded beams by using FSDBT and Hamilton's principle. ${ }^{10}$ The material properties are assumed to vary through the thickness according to the power-law method. Alshorbagy et al. investigated the free vibration analysis of functionally graded beams by using numerical finite elements method. ${ }^{11}$ The functionally graded beam is based on the Euler-Bernoulli beam theory and the equations of the motion are derived by using virtual work principle. The material properties are assumed to vary through the thickness and longitudinal direction according to the power-law. The effects of different boundary conditions are investigated. Demir and $\mathrm{Oz}$ investigated the resonance frequency behavior of a functionally graded beam under viscoelastic boundary conditions with the finite element method, by using Euler-Bernoulli beam theory. ${ }^{12}$ The material properties of the beam are supposed to vary through the thickness according to the power-law distribution. In order to attain different boundary conditions, various stiffness and damping coefficients are applied to viscoelastic support elements. Wattanasakulpong and Ungbhakorn investigated linear and nonlinear vibration responses of the functionally graded beams with the elastically restrained ends by taking into account the porosities which occur during fabrication. ${ }^{13}$ The differential transformation method (DTM) is used to solve linear and nonlinear vibration responses of FG beams. Duy et al. investigated free vibration response of a FG beam having an elastic foundation and spring supports. ${ }^{14}$ Young's modulus, mass density and width of the beam were supposed to vary in thickness and axial directions respectively following the exponential law. Shvartsman and Majak studied the buckling of axially functionally graded (FG) Euler-Bernoulli beams with elastically restrained ends. ${ }^{15}$ Calim examined the free and forced vibrations of AFG Timoshenko beams on the elastic/viscoelastic foundation by solving different kinds of problems. ${ }^{16}$ The vertical displacement of a beam is examined for various foundation parameters. Ravishankar et al. investigated the effects of different angular velocities and different aspect ratios of rotating and nonrotating hybrid composite beams, on the free vibration analysis of FGB. ${ }^{17}$ In their study, the free vibration analysis of rotating and non-rotating fiber metal laminate (FML) beams, hybrid composite beams (HCB), and functionally graded beams (FGB) are investigated. FML beams are high-performance hybrid structures based on alternating stacked arrangements of fiber-reinforced plastic (FRP) plies and metal alloy layers. Hybrid composite beams are materials that are made by adding two different fibers. Functionally graded beams are new materials that are designed to achieve a functional performance with gradually variable properties in one or more directions. The effects of different metal alloys, composite fibers, and different aspect ratios and angular velocities on the free vibration analysis of FML beams are studied. The effects of different an-

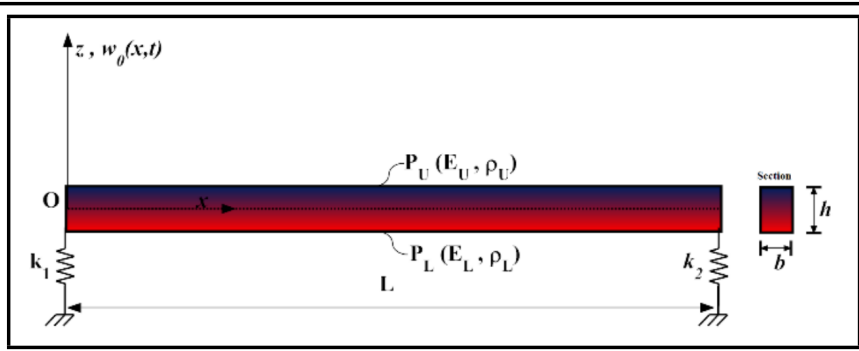

Figure 1. A spring supported FG beam.

gular velocities and different aspect ratios of rotating and nonrotating hybrid composite beams are also investigated. Finally, the effects of different angular velocities and different material distributions, namely the power law, exponential distribution, and Mori Tanaka's scheme on the free vibration analysis of FGB, are also investigated.

In this paper, the free and harmonic vibration analysis of a spring supported functionally graded beam is done by using finite element method. Timoshenko beam theory is considered for the given model. The material properties of the beam are assumed to vary through the thickness according to the powerlaw form. Various stiffness values are examined for the springsupport. These values provide the solution for free-free $(\kappa=0$, the support stiffness) and simply-supported $(\kappa=\infty)$ boundary conditions as well. In the numerical examples, the steady state responses to a sinusoidal varying force are determined for the support reactions in the frequency domain.

Lagrange equations are used in the finite element modelling. By using the Lagrange equations, the problem is reduced to a system of algebraic equations. The convergence study is based on the numerical values obtained for various sizes of an element. The accuracy of the results is established by comparison with previously published exact results of beams based on Timoshenko beam theory obtained for the special cases of the investigated problem. Results given in this paper may be useful for further investigations in this field. The support stiffness, volume fraction coefficient and ratio of Young module level effect on the frequency parameters and force transmissibility are investigated. For the low spring values, rigid body motion occurs in the spring-beam system, therefore volume fraction coefficient is limited on the natural frequencies.

\section{THEORY AND FORMULATIONS}

The spring point supported functionally graded beam of length $L$, thickness $h$, and width $b$, is considered as seen in Fig. 1. The dynamical behavior of the beam is governed by the Timoshenko beam theory and all the transverse deflections occur in the same plane, defined by the $x$ and $z$ axes. The origin of axis is chosen at the left end of the beam as shown in Fig. 1.

$E(z), G(z), \rho(z), \nu(z)$ are the material properties and elastic modulus, shear modulus, density and Poisson's ratio respectively. $E(z), G(z), \rho(z), \nu(z)$ of the functionally graded beam are assumed to vary through the thickness according to the power law distribution in Eq. (1); $P_{U}$ and $P_{L}$ are the corresponding material properties of the upper and lower surfaces of the beam and $\mathrm{n}$ is the power-law exponent. The variation of 


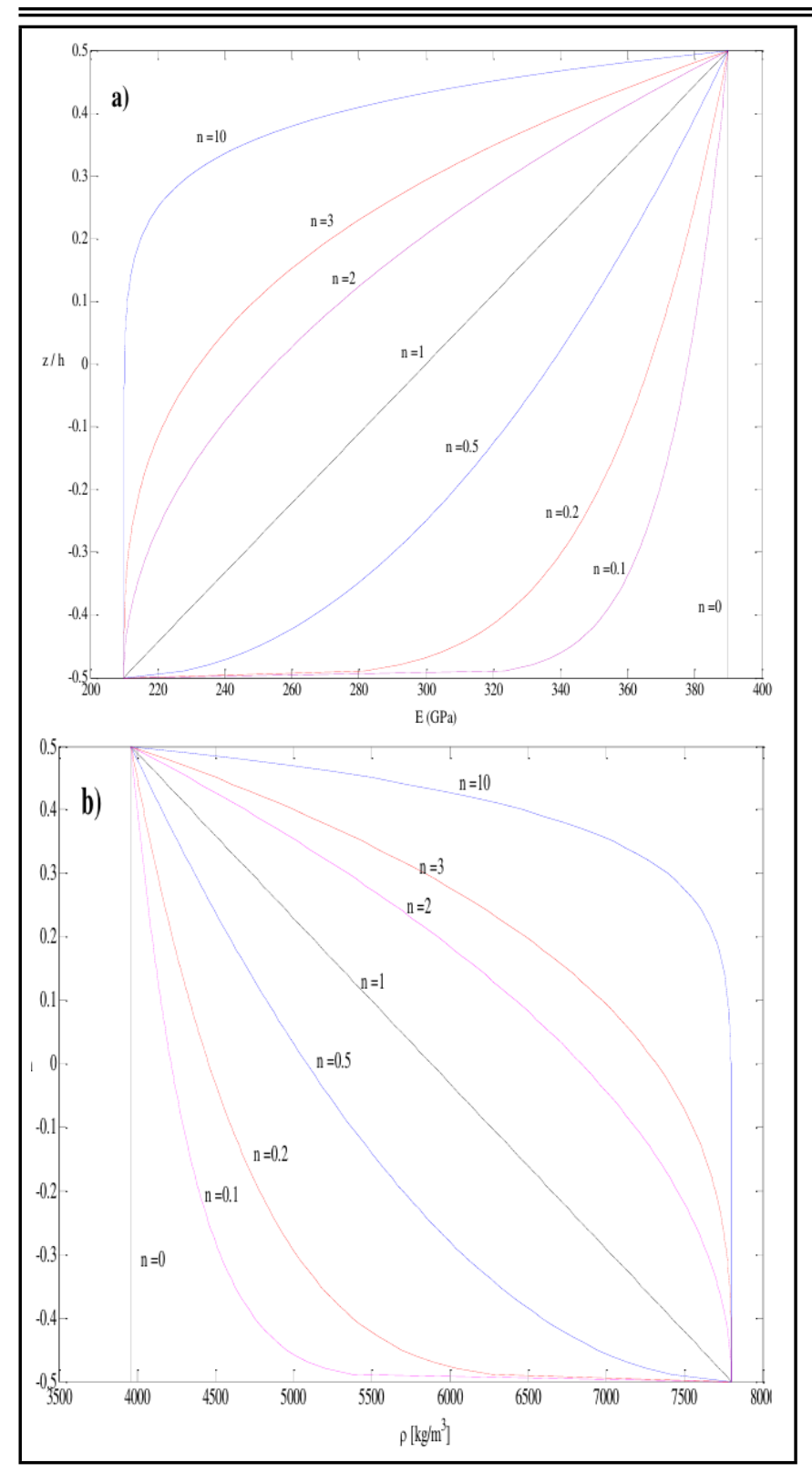

Figure 2. a) The variation of Young's modulus and b) the mass density in the thickness direction of the beam.

Young's modulus and the mass density in the thickness direction of the beam can be seen from Fig. 2.

$$
P(z)=\left(P_{U}-P_{L}\right)\left(\frac{z}{h}+\frac{1}{2}\right)^{n}+P_{L} .
$$

Lower surface of the beam is steel. Upper surface of the beam varies in conjunction with the change of $E_{\text {ratio. }}$ In other words, different material properties for the upper surface can be obtained by assigning different values to $E_{\text {ratio }}$. The material distribution changes continuously from the upper surface to the lower surface with respect to thickness and power-law exponent. Material properties $E$ and $\rho$ of the steel are $210 \mathrm{GPa}$ and $7800 \mathrm{~kg} / \mathrm{m}^{3}$ respectively. $E_{U}$ : Young's modulus of upper surface material, $E_{L}$ : Young's modulus of lower surface material, $\rho_{U}$ : density of upper surface material, $\rho_{L}$ : density of lower surface material.

The transverse displacement, $w(x, z, t)$, rotation of crosssection, $\theta(x, t)$, and axial displacement $u(x, z, t)$ of any point within the framework of Timoshenko beam theory are given as:

$$
\begin{gathered}
u(x, z, t)=u_{0}(x, t)-z \cdot \theta(x, t) ; \\
w(x, z, t)=w_{0}(x, t) .
\end{gathered}
$$

The strains and stresses of the beam are:

$$
\begin{gathered}
\varepsilon_{x x}=\frac{\partial u_{0}}{\partial x}-z \frac{\partial \theta}{\partial x} \\
\gamma_{x z}=\frac{\partial w}{\partial x}+\frac{\partial u}{\partial z}=\frac{\partial w_{0}}{\partial x}-\theta ; \\
\sigma_{x}=E(z) \cdot \varepsilon_{x} \\
\tau_{x z}=\kappa_{c} \cdot G(z) \cdot \gamma_{x z} .
\end{gathered}
$$

The elastic strain energy of a finite element obeying Hooke's law, can be written as follows after some arrangement:

$$
\begin{aligned}
& U=\frac{1}{2} \int_{0}^{L} \int_{A}\left(\sigma_{x x} \varepsilon_{x x}+\tau_{x z} \gamma_{x z}\right) d A d x= \\
& \frac{1}{2} \int_{0}^{L} \int_{A}\left(\varepsilon_{x x}^{2} E(z)+\gamma_{x z}^{2} \kappa_{c} G(z)\right) d A d x ; \\
& U=\frac{1}{2} \int_{0}^{L}\left[\begin{array}{l}
A_{x x}\left(\frac{\partial u_{0}}{\partial x}\right)^{2}-2 B_{x x}\left(\frac{\partial u_{0}}{\partial x} \frac{\partial \theta}{\partial x}\right) \\
\left.\quad+D_{x x}\left(\frac{\partial \theta}{\partial x}\right)^{2}+A_{x z}\left(\frac{\partial w_{0}}{\partial x}-\theta\right)^{2}\right] d x ;
\end{array}\right.
\end{aligned}
$$

where $A_{x x}$ axial, $D_{x x}$ bending, $A_{x z}$ shear and $B_{x x}$ coupling rigidity terms.

$$
\left(A_{x x}, B_{x x}, D_{x x}\right)=\int_{A} E(z)\left(1, z, z^{2}\right) d A ;
$$

$A_{x z}=\int_{A} \kappa_{c} G(z) d A=\kappa_{c}\left(G_{U}-G_{L}\right) \frac{b h}{n+1}+\kappa_{c} G_{L} b h$

Shear stress correction coefficient $\kappa_{c}$ is assumed $\kappa_{c}=\frac{5}{6}$ for rectangular section.

The kinetic energy of the finite element beam due to bending without the rotary inertia effects and axial displacement is given as:

$$
\begin{aligned}
T & =\frac{1}{2} \int_{0}^{L} \int_{A} \rho(z)\left(\dot{u}^{2}+\dot{w}^{2}\right) d A d x= \\
& \int_{0}^{L} \int_{A} \rho(z)\left[\left(\frac{\partial u_{0}}{\partial t}-z \frac{\partial \theta}{\partial t}\right)^{2}+\left(\frac{\partial w_{0}}{\partial t}\right)^{2}\right] d A d x ;
\end{aligned}
$$




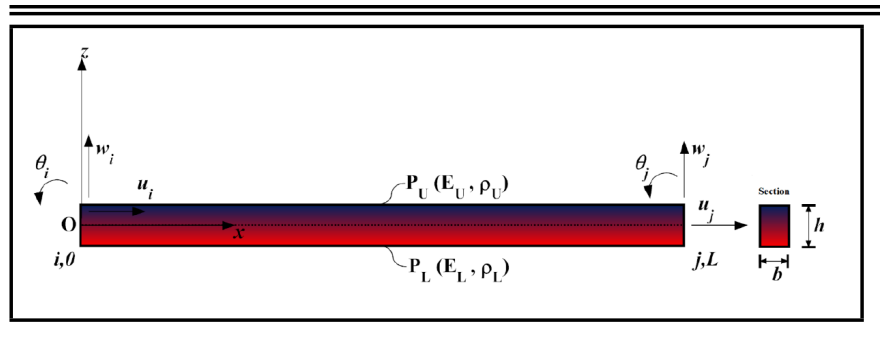

Figure 3. The degree of freedoms of FG beam finite element.

where $\dot{u}$ and $\dot{w}$ are the time derivatives of the axial and transverse displacement respectively Eq. (13) can be written as follow:

$$
\begin{aligned}
T=\frac{1}{2} \int_{0}^{L}\left[I_{A}\left(\frac{\partial u_{0}}{\partial t}\right)^{2}-2 I_{B}\left(\frac{\partial u_{0}}{\partial t} \frac{\partial \theta}{\partial t}\right)\right. \\
\left.+I_{D}\left(\frac{\partial \theta}{\partial t}\right)^{2}+I_{A}\left(\frac{\partial w_{0}}{\partial t}\right)^{2}\right] d x
\end{aligned}
$$

where $I_{A}, I_{B}, I_{D}$ are inertial terms as follow:

$$
\begin{gathered}
\left(I_{A}, I_{B}, I_{D}\right)=\int_{A} \rho(z)\left(1, z, z^{2}\right) d A \\
\beta=\frac{D_{x x}}{A_{x z}} .
\end{gathered}
$$

Figure 3 shows the six degrees of freedom for a two nodes finite element beam model. It has a length of $L$. The nodal displacements are given in Eq. (16):

$$
\{q\}=\left[u_{i}(t), w_{i}(t), \theta_{i}(t), u_{j}(t), w_{j}(t), \theta_{j}(t)\right]^{T} .
$$

The transverse, rotation and axial displacements of the element $w_{0}(x, t), \theta(x, t)$ and $u_{0}(x, t)$ are expressed in discrete form for using the nodal displacements by shape functions in matrix notation:

$$
\begin{gathered}
u_{0}(x, t)=[N]_{u_{0}}\{q\} ; \\
w_{0}(x, t)=[N]_{w_{0}}\{q\} ; \\
\theta(x, t)=[N]_{\theta}\{q\} ;
\end{gathered}
$$

$[N]_{u_{0}}$; The shape functions for the axial displacement and elements (see Eq. (20)); $[N]_{w_{0}}$; The shape functions for bending displacement (see Eq. (21)); $[N]_{\theta}$; The shape functions for the rotation of cross-section displacement (see Eq. (22)).

The discretized displacement Eqs. (17)-(19) are substituted in Eqs. (9)-(13) to obtain:

$$
\begin{aligned}
\mathrm{U}=\frac{1}{2} \int_{0}^{L}\left[A_{x x}\left(\left[N^{\prime}\right]_{u} \cdot\{q\}_{u}\right)^{2}\right. & \\
-2 B_{x x}\left(\left[N^{\prime}\right]_{u}\right. & \left.\cdot\{q\}_{u}\right)\left(\left[N^{\prime \prime}\right]_{w} \cdot\{q\}_{w}\right) \\
& \left.+D_{x x}\left(\left[N^{\prime \prime}\right]_{w} \cdot\{q\}_{w}\right)^{2}\right] d x
\end{aligned}
$$

$$
\begin{aligned}
\mathrm{T}=\frac{1}{2} \int_{0}^{L} & {\left[I_{A}\left([N]_{u} \cdot\{\dot{q}\}_{u}\right)^{2}\right.} \\
& -2 I_{B}\left([N]_{u} \cdot\{\dot{q}\}_{u}\right)\left(\left[N^{\prime}\right]_{w} \cdot\{\dot{q}\}_{w}\right) \\
& \left.+I_{A}\left([N]_{w} \cdot\{\dot{q}\}_{w}\right)^{2}+I_{D}\left(\left[N^{\prime}\right]_{w} \cdot\{\dot{q}\}_{w}\right)^{2}\right] d x .
\end{aligned}
$$

Expansion of Eqs. (23)-(24) are written as follow:

$$
U=\frac{1}{2}\{q\}^{T}\left[\begin{array}{c}
A_{x x} \int_{0}^{L}\left[N^{\prime}\right]_{u_{0}}^{T}\left[N^{\prime}\right]_{u_{0}} d x \\
-B_{x x} \int_{0}^{L}\left[N^{\prime}\right]_{u_{0}}{ }^{T}\left[N^{\prime}\right]_{\theta} d x \\
-B_{x x} \int_{0}^{L}\left[N^{\prime}\right]_{\theta}^{T}\left[N^{\prime}\right]_{u_{0}} d x \\
+D_{x x} \int_{0}^{L}\left[N^{\prime}\right]_{\theta}^{T}\left[N^{\prime}\right]_{\theta} d x \\
+A_{x z} \int_{0}^{L}\left[\left[N^{\prime}\right]_{w_{0}}-[N]_{\theta}\right]^{T} \\
\cdot\left[\left[N^{\prime}\right]_{w_{0}}-[N]_{\theta}\right] d x
\end{array}\right]\{q\}
$$

$$
T=\frac{1}{2}\{\dot{q}\}^{T}\left[\begin{array}{l}
I_{A} \int_{0}^{L}[N]_{u_{0}}{ }^{T}[N]_{u_{0}} d x \\
-I_{B} \int_{0}^{L}[N]_{u_{0}}{ }^{T}[N]_{\theta} d x \\
-I_{B} \int_{0}^{L}[N]_{\theta}^{T}[N]_{u_{0}} d x \\
+I_{D} \int_{0}^{L}[N]_{\theta}^{T}[N]_{\theta} d x \\
+I_{A} \int_{0}^{L}[N]_{w_{0}}{ }^{T}[N]_{w_{0}} d x
\end{array}\right]\{\dot{q}\} .
$$

Terms in Eqs. (25) and (26) between brackets consist of the stiffness and mass matrices of an element. The functional of the finite element is:

$$
I=T-U .
$$

By using the Lagrange equations:

$$
\frac{\partial I}{\partial q_{k}}-\frac{\mathrm{d}}{\mathrm{d} t} \frac{\partial I}{\partial \dot{q}_{k}}=0 ; \quad k=1,2,3,4,5,6 .
$$

Equation (28) yields to the following equation for the considered element in matrices form:

$$
\left[K_{e}\right]\{q\}+\left[M_{e}\right]\{\ddot{q}\}=0 .
$$

The matrices $\left[K_{e}\right],\left[M_{e}\right]$ are $6 \times 6$ element stiffness and mass matrices respectively.

Having developed formulations for the mass matrix and stiffness matrix of a finite element, the global equations for a finite element model of a structure can be assembled and the functional of the problem in terms of the kinetic energy of the beam, potential energy of the beam, potential energy of the external forces and couples, potential energy of the supports at any time can be written as follows:

$$
T=\frac{1}{2}\{\dot{q}\}^{T}[M]\{\dot{q}\} ;
$$




$$
\begin{gathered}
{[N]_{u_{0}}=\left[\begin{array}{lllllll}
1-\frac{x}{L} & 0 & 0 & \frac{x}{L} & 0 & 0
\end{array}\right] ;} \\
{[N]_{w_{0}}=\left[\begin{array}{llllll}
0 & 1-\frac{x\left(12 \beta-2 x^{2}\right)+3 L x^{2}}{L\left(L^{2}+12 \beta\right)} & \frac{x(L-x)\left(L^{2}-x L+6 \beta\right)}{L\left(L^{2}+12 \beta\right)} & 0 & \frac{x\left(12 \beta-2 x^{2}\right)+3 L x^{2}}{L\left(L^{2}+12 \beta\right)} & \frac{-x(6 \beta+L x)(L-x)}{L\left(L^{2}+12 \beta\right)}
\end{array}\right] ;} \\
{[N]_{\theta}=\left[\begin{array}{llllll}
0 & \frac{-6 x(L-x)}{L\left(L^{2}+12 \beta\right)} & \frac{L-x}{L}-\frac{3 x(L-x)}{\left(L^{2}+12 \beta\right)} & 0 & \frac{6 x(L-x)}{L\left(L^{2}+12 \beta\right)} & \frac{x\left(-2 L^{2}+3 x L+12 \beta\right)}{L\left(L^{2}+12 \beta\right)}
\end{array}\right] .}
\end{gathered}
$$

Table 1. Non-dimensional frequencies of a simply supported beam.

\begin{tabular}{|l|c|c|c|c|c|c|c|c||}
\hline$L / h=5$ & $n=0$ & $n=0.2$ & $n=0.5$ & $n=1$ & $n=2$ & $n=5$ & $n=10$ & $n=\infty$ \\
\hline Simsek $^{18}$ & 5.15247 & 4.80657 & 4.4083 & 3.99023 & 3.63438 & 3.43119 & 3.31343 & 2.67718 \\
\hline Present & 5.15249 & 4.80541 & 4.40791 & 3.99026 & 3.63441 & 3.43122 & 3.31345 & 2.67719 \\
\hline Difference\% & $0.00 \%$ & $0.00 \%$ & $0.00 \%$ & $0.00 \%$ & $0.00 \%$ & $0.00 \%$ & $0.00 \%$ & $0.00 \%$ \\
\hline$L / h=20$ & $n=0$ & $n=0.2$ & $n=0.5$ & $n=1$ & $n=2$ & $n=5$ & $n=10$ & $n=\infty$ \\
\hline Simsek $^{18}$ & 5.46032 & 5.08265 & 4.65137 & 4.20505 & 3.83676 & 3.65088 & 3.54156 & 2.83713 \\
\hline Present & 5.46032 & 5.08134 & 4.65092 & 4.20507 & 3.83678 & 3.6509 & 3.54157 & 2.83714 \\
\hline
\end{tabular}

Table 2. First non-dimensional frequency parameters $\lambda_{1}$ of simply supported FG beam for different material 1 distribution and different $E_{\text {ratio }}$.

\begin{tabular}{||l|c|c|c|c|c|c|c||}
\hline$E_{r}$ & $n=0$ & $n=0.2$ & $n=0.5$ & $n=1$ & $n=2$ & $n=5$ & $n=10$ \\
\hline 0.25 & 2.216769 & 2.370706 & 2.457361 & 2.700119 & 2.801825 & 2.858641 & 3.004167 \\
\hline 0.5 & 2.636197 & 2.70634 & 2.753144 & 2.890151 & 2.941729 & 2.970507 & 3.051721 \\
\hline 1 & 3.134986 & 3.134986 & 3.134986 & 3.134986 & 3.134986 & 3.134986 & 3.134986 \\
\hline 2 & 3.728146 & 3.671495 & 3.624319 & 3.43699 & 3.371294 & 3.344659 & 3.266996 \\
\hline 3 & 4.125872 & 4.040858 & 3.967664 & 3.648257 & 3.525877 & 3.480658 & 3.367992 \\
\hline 4 & 4.433537 & 4.329876 & 4.239048 & 3.818545 & 3.643939 & 3.581012 & 3.448407 \\
\hline
\end{tabular}

$$
\begin{gathered}
U=\frac{1}{2}\{q\}^{T}[K]\{q\} ; \\
V=-\{q\}^{T}\{F\} ; \\
V_{s}=\frac{1}{2} k_{1}\left[q_{1}(t)\right]^{2}+\frac{1}{2} k_{2}\left[q_{p-1}(t)\right]^{2} ; \\
p=3 m+3 ; \quad n=m+1 ;
\end{gathered}
$$

where $m$ is the number of the finite elements, $n$ is the number of the nodes of the system, $p$ is the total number of degrees of freedom of the model, $k_{1}, k_{2}$ represent the stiffness of the first and second support respectively.

Functional for the whole system can be expressed as:

$$
I=T-\left(U+V+V_{s}\right) ;
$$

then, using the Lagrange equations for the whole system:

$$
\frac{\partial I}{\partial q_{k}}-\frac{\mathrm{d}}{\mathrm{d} t} \frac{\partial I}{\partial \dot{q}_{k}}=0 ; \quad k=1, p ;
$$

yields the following equation for the whole system:

$$
[K]\{q\}+\left[K_{s}\right]\{q\}+[M]\{\ddot{q}\}=\{F(t)\} ;
$$

where the matrixes $[K],\left[K_{s}\right],[M]$ are $p \times p$ system stiffness matrix, support stiffness matrix and the mass matrix respectively. The only nonzero terms of the $\left[K_{s}\right]$ matrix are $K_{s 22}$, $K_{s(p-1)(p-1)}$.

Considering that the concentrated force affects the midpoint of the beam, it is expressed as:

$$
\{F(t)\}=\{Q\} \cdot e^{i \omega t} ; \quad Q_{\left(3 \frac{m}{2}+2\right)} \neq 0 .
$$

The time-dependent nodal displacements can be expressed as follows:

$$
\{q(t)\}=\{\bar{q}\} e^{i \omega t}
$$

in Eq. (39), the elements of $\{\bar{q}\}$ are complex variables containing a phase angle. By taking into account Eqs. (37)- (39) can be expressed in the following matrix form:

$$
[K]\{\bar{q}\}+\left[K_{s}\right]\{\bar{q}\}-\omega^{2}[M]\{\bar{q}\}=\{Q\} .
$$

Equation (40) can be written in the following form:

$$
\left([K]+\left[K_{s}\right]-\omega^{2}[M]\right)\{\bar{q}\}=\{Q\} .
$$

The maximum total magnitude of the reaction forces of the supports is given as follow:

$$
P=\left(k_{1}\right) \bar{q}_{1}+\left(k_{2}\right) \bar{q}_{p-1}
$$

therefore the force transmissibility at the supports is determined by:

$$
T_{R}=\frac{P}{Q}
$$

$T_{R}$ : Force transmissibility.

\section{NUMERICAL RESULTS}

The transmissibility and natural frequency parameters of a functionally graded beam, spring supported at the ends is calculated numerically. The ratio of the beam of length $L$ to thickness $h(L / h)$ is assumed 20 for brevity. The parameters $k_{i}$ are taken as having the same respective values at the two supports denoted by $k_{s}=k_{1}=k_{2}$. $\kappa$ dimensionless stiffness coefficient and $\lambda$ frequency parameter are defined as follows:

$$
\kappa=\frac{k_{s} L^{3}}{E I} ; \quad \lambda=\omega L^{2} \sqrt{\frac{\rho_{L} A}{E_{L} I}} .
$$

Equation (41) can be written by using Eq. (44) in the following dimensionless form:

$$
\left\{[K]+\kappa\left[K_{s}\right]-\lambda^{2}[M]\right\}\{\bar{q}\}=\{Q\} .
$$


Table 3. Second non-dimensional frequency parameters $\lambda_{2}$ of simply supported FG beam for different material distribution and different E ratio.

\begin{tabular}{||l|c|c|c|c|c|c|c||}
\hline \hline$E_{r}$ & $n=0$ & $n=0.2$ & $n=0.5$ & $n=1$ & $n=2$ & $n=5$ & $n=10$ \\
\hline 0.25 & 4.406245 & 4.711416 & 4.883838 & 5.369031 & 5.572323 & 5.685525 & 5.973866 \\
\hline 0.5 & 5.239939 & 5.378873 & 5.471799 & 5.745394 & 5.848865 & 5.906397 & 6.067487 \\
\hline 1 & 6.231373 & 6.231373 & 6.231373 & 6.231373 & 6.231373 & 6.231373 & 6.231373 \\
\hline 2 & 7.410393 & 7.298264 & 7.204812 & 6.832463 & 6.700543 & 6.64662 & 6.491215 \\
\hline 3 & 8.200948 & 8.032693 & 7.887747 & 7.253505 & 7.008435 & 6.916868 & 6.690143 \\
\hline 4 & 8.812491 & 8.607338 & 8.427495 & 7.592957 & 7.243986 & 7.116811 & 6.848703 \\
\hline
\end{tabular}

Table 4. Third non-dimensional frequency parameters $\lambda_{3}$ of simply supported FG beam for different material 3distribution and different E ratio.

\begin{tabular}{|l|c|c|c|c|c|c|c|}
\hline$E_{r}$ & $n=0$ & $n=0.2$ & $n=0.5$ & $n=1$ & $n=2$ & $n=5$ & $n=10$ \\
\hline 0.25 & 6.544575 & 6.996 & 7.252524 & 7.979299 & 8.283845 & 8.452594 & 8.878737 \\
\hline 0.5 & 7.782856 & 7.988103 & 8.125884 & 8.535158 & 8.691026 & 8.777235 & 9.015712 \\
\hline 1 & 9.255427 & 9.255427 & 9.255427 & 9.255427 & 9.255427 & 9.255427 & 9.255427 \\
\hline 2 & 11.00662 & 10.841163 & 10.7031 & 10.150071 & 9.95107 & 9.868732 & 9.635548 \\
\hline 3 & 12.180827 & 11.932586 & 11.718537 & 10.778007 & 10.409854 & 10.269999 & 9.926882 \\
\hline 4 & 13.089151 & 12.786489 & 12.520962 & 11.284432 & 10.76176 & 10.568081 & 10.159513 \\
\hline
\end{tabular}
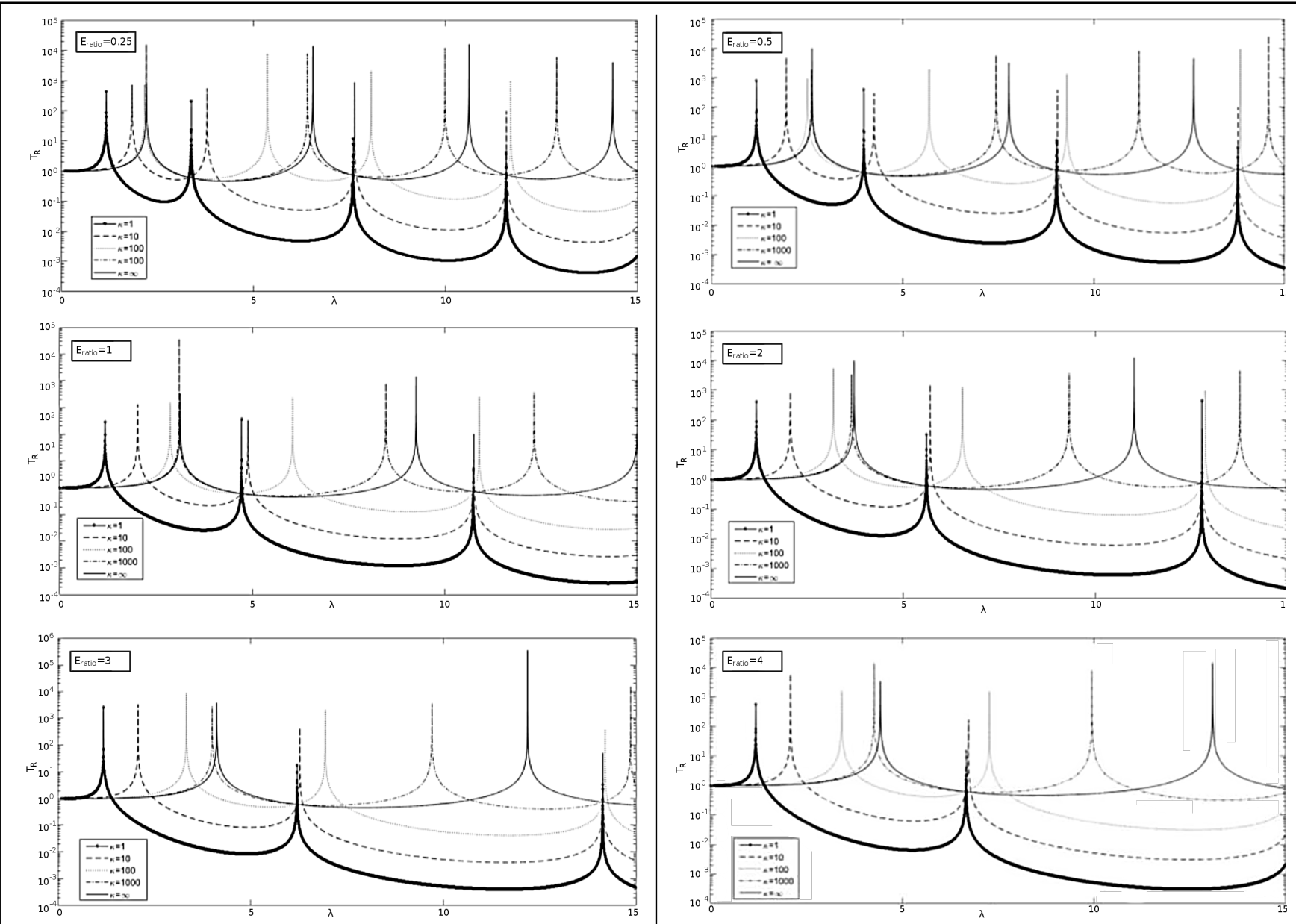

Figure 4. The force transmissibility of the FG beam for the various $\kappa, E_{\text {ratio }}=0.25,0.5,1,2,3,4$ and $n=0$.

The eigenvalues $\lambda$ are found from the condition that the determinant of linear homogeneous equations given by Eq. (45) by neglecting the force.

Young module ratio can be written as follow:

$$
E_{\text {ratio }}=\frac{E_{U}}{E_{L}}
$$

and the mass ratio is considered as constant:

$$
\rho_{\text {ratio }}=\frac{\rho_{U}}{\rho_{L}}=1 \text {. }
$$

The boundary condition of a simply supported beam is represented with infinite lateral support stiffness $\kappa=\infty$ at the supports for comparing the obtained results with the existing results. The dimensionless frequency parameters are compared with Simsek ${ }^{18}$ at Table 1 for $L / h=5,20$ and various $n$ values. The obtained values show excellent agreement with those of Simsek. $^{18}$

Firstly, the first three natural frequencies of simply supported FG Timoshenko beam with $L / h=20$ are calculated in Tables 2-4. The variation of fundamental non-dimensional 


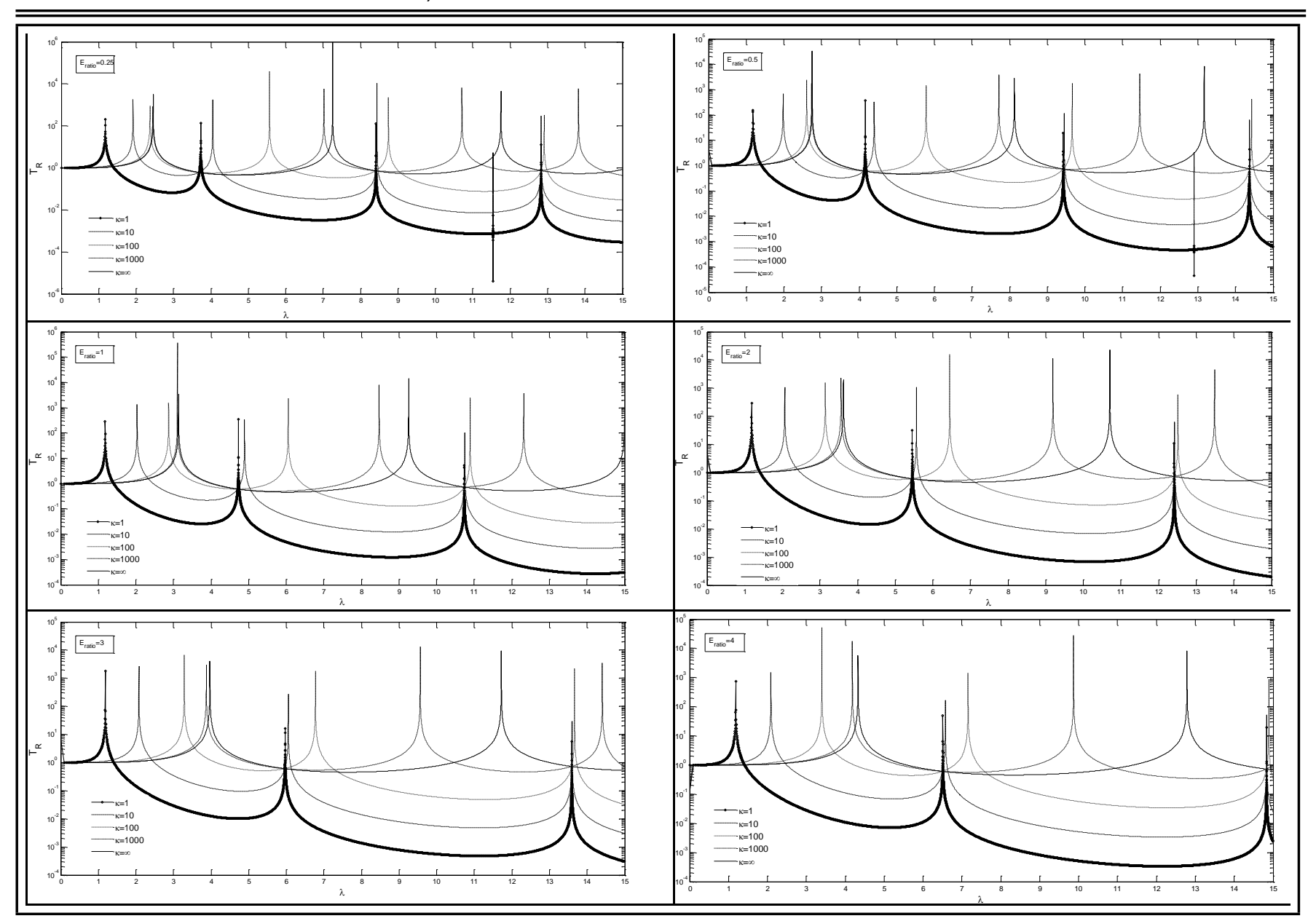

Figure 5. The force transmissibility of the FG beam for the various $\kappa, E_{\text {ratio }}=0.25,0.5,1,2,3,4$ and $n=0.5$.

frequencies with different $E_{\text {ratio }}$ and power-law exponent ' $n$ ' for simply supported beam can be observed. The mass ratio is considered as in Eq. (47).

The first three natural frequencies are also obtained for the harmonic analysis of the spring supported beam situation. Because of the symmetry of the structure and external force in the harmonic analysis of the spring supported beam, only symmetrical vibrations with respect to the midpoint of the beam arise. The symbol $S$ represents symmetrical vibration with respect to the midpoint of the beam. Although the $S-1$ mode existing in the case of simply supported beam does not occur in the completely free beam, it occurs for every value of stiffness parameter, which is different from zero. Therefore, the stiffness parameters vary from 1 to $10^{8}$. While increasing the stiffness parameter $\kappa$, the frequency parameters become the values of a simply supported beam. For the lower values of stiffness parameter $\kappa$, since the two supports have the same respective values, the beam vibrates in vertical rigid body modes ( $\mathrm{Z}$ direction) in addition to elastic modes. This condition can be defined as "While the coefficient of the spring of the support increases, the vibration mode goes from rigid to elastic". Namely, for the low values of spring coefficient $(\kappa=1$ and $\kappa=10$ ), beam vibrates predominantly in rigid body mode, and for the high values of spring coefficient (simply supported condition), beam vibrates predominantly in elastic body mode. This situation affects the influence of the $E_{\text {ratio }}$ and power-law exponent ' $n$ ' on the frequency parameters.
According to Tables 2-4, natural frequency behavior of simply supported FG beam is very consistent in each mode. When $E_{\text {ratio }} \leq 1$, dimensionless natural frequency parameters increase while $\mathrm{n}$ increases from 0 to 10 (Tables 2-4). However, an opposite situation is observed when $E_{\text {ratio }}>1$. Dimensionless natural frequency parameters decrease while $n$ increases (Tables 2-4). Thus, both conditions approach to homogenous beam condition. It can be observed in Tables 2-4 that the variation of $E_{\text {ratio }}$ is more effective on the frequency parameters than the variation of the $n$ for simply supported FG beams.

It can be observed in any figure of Figs. 4-8 that the frequency parameters and the difference between frequencies of each $n$, increase with increasing $\kappa$ when $\kappa>1$. Since rigid modes are dominant for $1<\kappa<10$, n doesn't cause a significant change in frequencies for these boundary conditions. This can be observed from the differences between frequencies of each n (Figs. 4-8).

When considering elastic supported FG beams, effect of $E_{\text {ratio }}$ is important at small $n$ values (Figs. 4-8). When $E_{\text {ratio }}<1$, dimensionless natural frequencies of FG beam increase with increasing $n$. When $E_{\text {ratio }}>1$, dimensionless natural frequency values decrease with increasing $n$ (Figs. 48).

At high n values, difference between each $E_{\text {ratio }}$ values is smaller than those of small $n$ values (Figs. 4-8).

With increasing $E_{\text {ratio }}$ the differences between natural fre- 


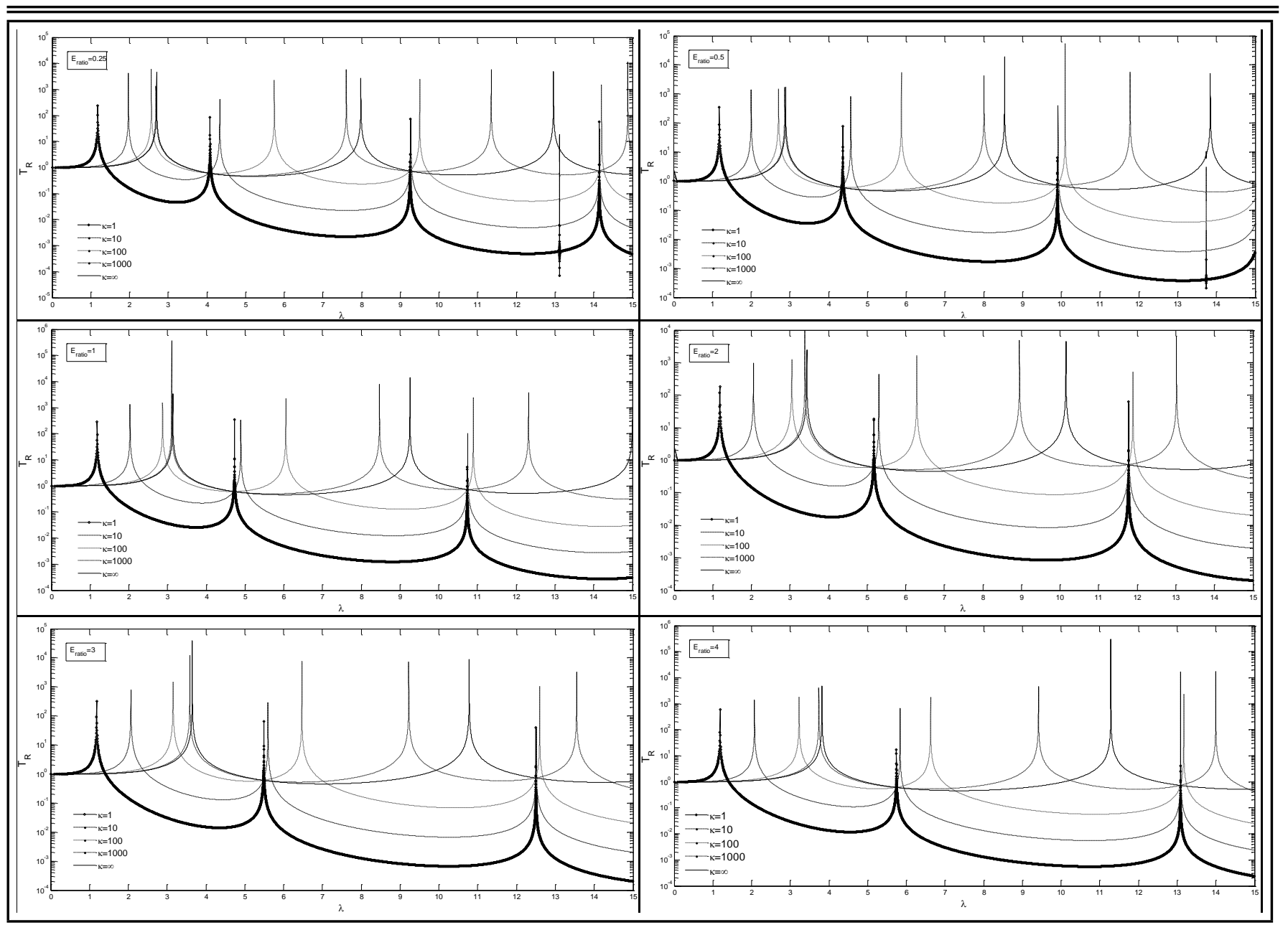

Figure 6. The force transmissibility of the FG beam for the various $\kappa, E_{\text {ratio }}=0.25,0.5,1,2,3,4$ and $n=2$.

quencies are increasing except for $\kappa=1$ and $\kappa=10$. The natural frequencies increase with increasing $E_{\text {ratio }}$ and the effect of increasing $E_{\text {ratio }}$ increases with increasing $\kappa$ (any figure of Figs. 4-8).

The effect of increasing power-law exponent " $n$ " is lighter on the first resonance frequency $(S-1)$ than the others, especially at the $\kappa=1$ and $\kappa=10$ spring coefficient (Figs. 4-8).

With getting the higher frequencies; the frequency differences of the values of $\kappa=1,10,100$ decrease. This can be seen in Figs. 4-8.

With getting the higher frequencies; the frequency differences of the values of $\kappa=1000, \infty$ increase. This can be seen in Figs. 4-8.

Because of the influence of the rigid body mode $(\kappa=1,10)$, the effect of the power exponent coefficient is limited. With the increasing coefficient of the stiffness of spring, the effect of the power exponent coefficient increases. So the maximum effect can be seen at the simply supported condition where $\kappa=\infty$. This can be seen in Figs. 4-8.

There is an optimum value of force transmissibility at $\kappa=1$, the intersection point of these lines exist at $\kappa=1$, through which all the response curves pass except first natural frequencies, regardless of the power-law exponent " $n$ ". This can be seen in Figs. 4-8.

The variation of the $E_{\text {ratio }}$ is more effective on the natural frequency than the variation in power exponent $(n)$. This can be seen in Figs. 4-8.

\section{CONCLUSION}

The harmonic vibration analysis of functionally graded spring supported beam is investigated by means of finite element method. Various stiffness values are examined for the spring-support. The equations of motion are derived using Lagrange equations under the assumptions of the Timoshenko beam theory. The material properties of the beam vary trough the thickness of the beam according to the power-law volume fraction function.

According to the numerical results:

- Only the symmetrical vibrations with respect to the midpoint of the beam arise because of the symmetry of the structure and external force in the harmonic analysis of the spring supported beam.

- The effect of the power exponent coefficient is limited for the lower stiffness coefficient, because of the influence of the rigid body mode. The effect of the power exponent coefficient increases with the increasing coefficient of the stiffness of spring.

- There is an optimum value of force transmissibility at $\kappa=1$, The optimum value is the point through which 


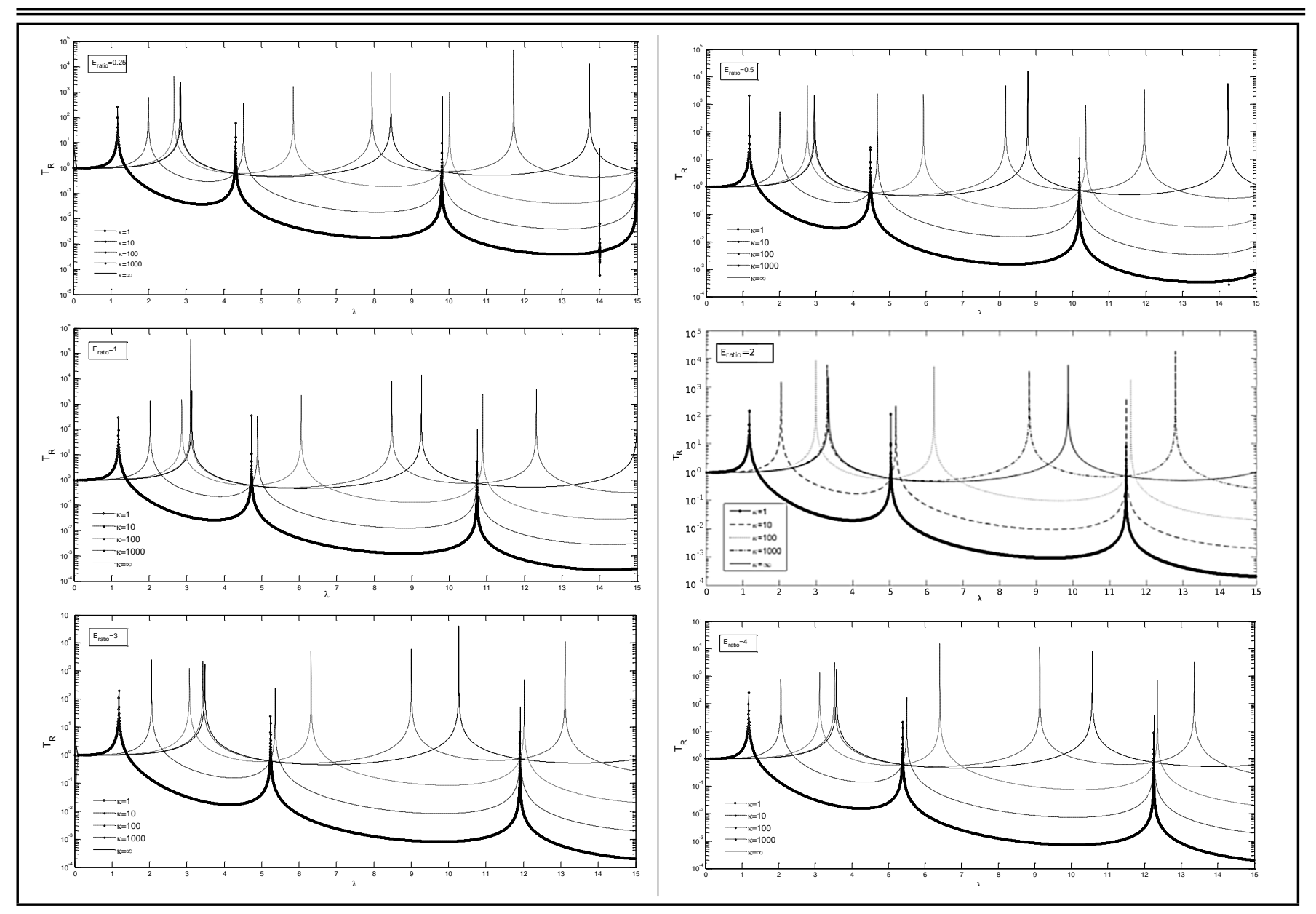

Figure 7. The force transmissibility of the FG beam for the various $\kappa, E_{\text {ratio }}=0.25,0.5,1,2,3,4$ and $n=5$.

all the response curves pass, regardless of the power-law exponent " $n$ " except first natural frequencies.

- The variation of the $E_{\text {ratio }}$ is more effective on the natural frequency than the variation in power exponent $(n)$.

- The study concludes that the support stiffness, volume fraction coefficient and ratio of young module level all strongly affect the natural frequencies and the force transmissibility except the rigid body modes.

\section{REFERENCES}

1 Sankar, B. V. An elasticity solution for functionally graded beams, Composites Science and Technology, 61(689), 96, (2001). https://dx.doi.org/10.1016/s0266-3538(01)000070 .

2 Aydogdu, M. and Taskin, V. Free vibration analysis of functionally graded beams with simply supported edges, Mater Des, 28(5), 1651-1656, (2006). https://dx.doi.org/10.1016/j.matdes.2006.02.007.

3 Simsek, M. and Kocaturk, T. Free and forced vibration of a functionally graded beam subjected to a concentrated moving harmonic load, Composite Structures, 90(4), 465-473, (2009). https://dx.doi.org/10.1016/j.compstruct.2009.04.024.
4 Su, H., Banerjee J. R. and Cheung C. W. Dynamic stiffness formulation and free vibration analysis of functionally graded beams, Composite Structures, 106, 854-862, (2013). https://dx.doi.org/10.1016/j.compstruct.2013.06.029.

5 Suddoung, K., Charoensuk, J. and Wattanasakulpong N. Vibration response of stepped FGM beams with elastically end constraints using differential transformation method, Appl. Acoust., 77, 20-28, (2014). https://dx.doi.org/10.1016/j.apacoust.2013.09.018.

${ }^{6} \mathrm{Li}, \mathrm{X}$. A unified approach for analyzing static and dynamic behaviors of functionally graded Timoshenko and Euler Bernoulli beams, J. Sound. Vib., 318(3-4), 1210-1229, (2008). https://dx.doi.org/10.1016/j.jsv.2008.04.056.

7 Zhong, Z. and Yu, T. Analytical solution of a cantilever functionally graded beam. Compos. Sci. Technol., 67, 481-488, (2007). https://dx.doi.org/10.1016/j.compscitech.2006.08.023.

8 Kapuria, S., Bhattacharyya, M. and Kumar A. N. Bending and free vibration response of layered functionally graded beams: Atheoretical model and its experimental validation, Compos. Struct., 82, 390-402, (2008). https://dx.doi.org/10.1016/j.compstruct.2007.01.019.

9 Piovan, M. T. and Sampaio, R. A study on the dynamics of rotating beams with functionally graded prop- 


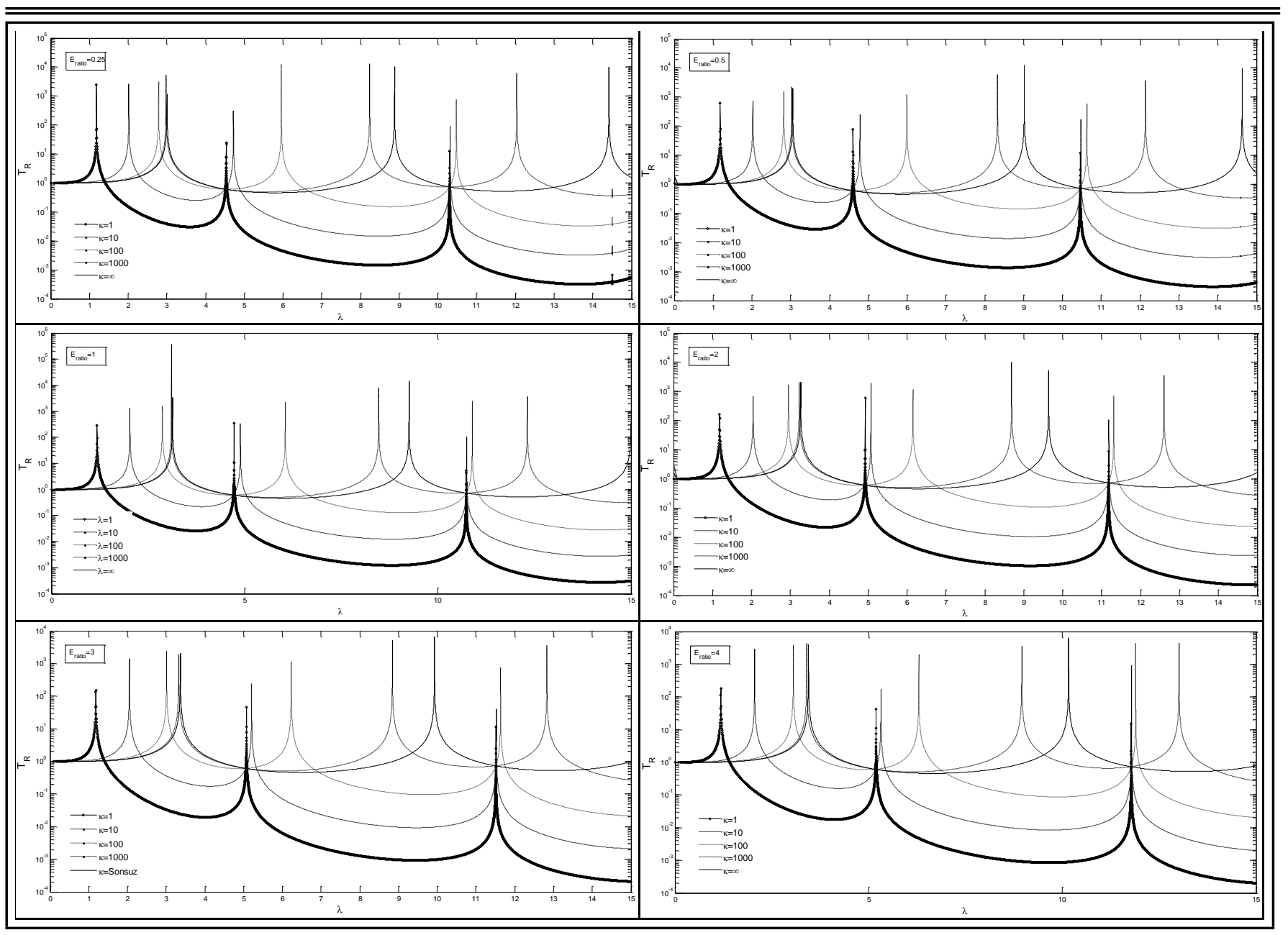

Figure 8. The force transmissibility of the FG beam for the various $\kappa, E_{\text {ratio }}=0.25,0.5,1,2,3,4$ and $n=10$.

erties. J. Sound. Vib., 327(1-2), 134-143, (2009). https://dx.doi.org/10.1016/j.jsv.2009.06.015.

10 Sina, S. A., Navazi, H. M. and Haddadpour, H. An analytical method for free vibration analysis of functionally graded beams, Mater. Des., 30, 741-747, (2009). https://dx.doi.org/10.1016/j.matdes.2008.05.015.

11 Alshorbagy, A. E., Eltaher, M. A. and Mahmoud, F. F. Free vibration characteristics of a functionally graded beam by finite element method, Appl. Math. Model., 35, 412-425 (2011). https://dx.doi.org/10.1016/j.apm.2010.07.006.

12 Demir C. and Oz, F. E. Free vibration analysis of a functionally graded viscoelastic supported beam, J. Vib. Control., 20, 2464-286, (2014). https://dx.doi.org/10.1177/1077546313479634.

13 Wattanasakulpong, N. and Ungbhakorn, V. Linear and nonlinear vibration analysis of elastically restrained ends FGM beams with porosities, Aerosp. Sci. Technol., 32(1), 111120, (2014). https://dx.doi.org/10.1016/j.ast.2013.12.002.

14 Duy, T. H., Van, T. N. and Noh, H. C. Eigen analysis of functionally graded beams with variable cross-section resting on elastic supports and elastic foundation, Structural Engineering and Mechanics, 52(5), 1033-1049, (2014). https://dx.doi.org/10.12989/sem.2014.52.5.1033.
15 Shvartsman, B. and Majak, J. Numerical method for stability analysis of functionally graded beams on elastic foundation, Appl. Math. Model, 40, 3713-3719, (2016). https://dx.doi.org/10.1016/j.apm.2015.09.060.

16 Calim, F. F. Free and forced vibration analysis of axially functionally graded Timoshenko beams on two-parameter viscoelastic foundation, Compos. Eng. Part B, 103, 98-112, (2016). https://dx.doi.org/10.1016/j.compositesb.2016.08.008.

17 Ravishankar, H., Rengarajan, R., Devarajan, K. and Kaimal, B. Free Vibration Bahaviour of Fiber Metal Laminates, Hybrid Composites and Functionally Graded Beams using Finite Element Analysis, International Journal of Acoustics and Vibration, 21(4), (2016). https://dx.doi.org/10.20855/ijav.2016.21.4436.

18 Simsek, M., Fundamental Frequency Analysis of Functionally Graded Beams by Using Different Higher-order Beam Theories, Nuclear Engineering and Design, 240, 697-705, (2010). https://dx.doi.org/10.1016/j.nucengdes.2009.12.013. 